\title{
Perceived social benefits versus perceived harms of smoking among Indonesian boys aged 12-16 years: A secondary analysis of Global Youth Tobacco Survey 2014
}

\author{
Nurul Kodriati', Elli Nur Hayati' ${ }^{2}$ Ailiana Santosa ${ }^{3}$, Lisa Purse/l'
}

\begin{abstract}
INTRODUCTION Smoking among boys has not been prioritised as a gender issue despite its high prevalence worldwide. In Indonesia, steep increases in prevalence have been observed in adolescent boys. This study explored how smoking-related beliefs are associated with smoking among this group.

METHODS Data extracted from the Global Youth Tobacco Survey Indonesia 2014, provided a nationally representative sample of 2729 male students aged 12-16 years. Measures of smoking-related beliefs were derived from eight survey items using principal component analysis. Associations between resulting components and smoking outcomes were modelled using logistic regression.

RESULTS Smoking prevalence was found to be almost tripling between ages 12 to 16 years. Smoking-related belief items clustered into two components: perceived social benefits and perceived harms. The four beliefs representing smoking's perceived social benefits and measures of smokers in the boys' social circles increased with age while the four beliefs representing smoking's perceived harms remained stable except an item of safe to smoke for one or two years, which increased with age. The two components of smoking-related beliefs were associated with smoking in opposite ways that represent boys' masculine tendency for risk-taking and risk minimisation. For example, score increases for perceived benefits were positively associated with susceptibility to future tobacco use (OR=1.6; 95\% CI: 1.3-1.9) but an increased score of perceived harm was negatively associated with susceptibility to future tobacco use $(\mathrm{OR}=0.8 ; 95 \% \mathrm{CI}$ : $0.7-0.9)$.

CONCLUSIONS Indonesian boys experience a rapid increase in smoking outcomes and smoking reported among their social circle. The sustained high percentage of smoking harms but also increased social benefits are similar to the concept of risk minimisation that is closely related to the masculine tendency to undermine health hazards of tobacco. Therefore, it is important to focus on these highly gender-related issues within the country.
\end{abstract}

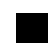

\section{AFFILIATION}

1 School of Health Sciences, National University of Ireland Galway, Galway, Ireland

2 Faculty of Psychology, University of Ahmad Dahlan, Yogyakarta,

Indonesia

3 Department of Public Health and Community Medicine, Institute of Medicine, Gothenburg University, Gothenburg, Sweden

CORRESPONDENCE TO

Nurul Kodriati. School of Health Sciences, National University of Ireland Galway, Galway, Ireland. E-mail: n.kodriati1@nuigalway.ie ORCID ID: https://orcid.org/00000003-2417-3590

KEYWORDS

smoking, adolescents, Indonesia, masculinity, smoking-related beliefs

Received: 3 June 2019

Revised: 20 November 2019

Accepted: 3 December 2019

\section{INTRODUCTION}

World-wide smoking prevalence among boys is higher than for girls ${ }^{1,2}$. In Indonesia, smoking prevalence in 2015 , dominated by male smokers, was ranked third in the world ${ }^{1}$. The majority of Indonesian male smokers initiated smoking at an age 10-19 years $^{3}$, with the prevalence in this age group increasing ${ }^{1,4}$ by $9.1 \%$ from 1990 to $2015^{1}$. The high prevalence among males $(27.7 \%)$ relative to females $(2.3 \%)^{1}$ and the young age of initiation led to our focus in the current study on smoking among adolescent boys.

Smoking has long been associated with 
masculinity in Indonesian culture and can be traced back to the colonial influences in Dutch society of the 17 th century ${ }^{5}$. Men's beliefs about their gender identity remain associated with their smoking behaviours. Among some boys, smoking is perceived to project an image that is very different from that of women, a phenomenon that is enhanced when there are cultural prohibitions on women smoking ${ }^{6}$. This link has been captured and strengthened by advertisements ${ }^{7}$ and popular media ${ }^{8}$, contributing to an environment that promotes smoking among boys. According to the Global Youth Tobacco Survey (GYTS) 2014, percentages of Indonesian boys who noticed tobacco advertisements or promotions at point-of-sale and were offered a free tobacco product from a tobacco company representative were $54.2 \%$ and $12.6 \%$, respectively ${ }^{8}$. These percentages indicate weak legislation on tobacco advertisements through various media such as TV, billboards, print media, and during events sponsored by the tobacco industry ${ }^{7}$. The legislation prohibits direct advertisement that could be circumvented by the tobacco industry by advertising smoking as an accepted lifestyle to enhance masculinity, to support friendship, and for enjoyment ${ }^{7,9}$.

Indonesian boys are further disadvantaged by the masculine social construction, which encourages them to have and exhibit characteristics such as being strong, tough, and heroic ${ }^{10}$. These are characteristics of Connell's hegemonic masculinity ${ }^{11}$. A type of masculinity that is mostly used in studying smoking behaviours among men ${ }^{12}$. Practised masculinities vary ${ }^{11}$ throughout a man's course of life, as they interplay with other social determinants of health, such as race or class, as described by the theoretical framework of Health Illness Men and Masculinities ${ }^{13}$. Such masculine characteristics often lead to risky and unhealthy behaviours, like smoking and alcoholism, among boys ${ }^{14}$. It is argued that the articulation of the complexity of masculinities and their relation to smoking has the potential to provide more understanding of the sustained high smoking prevalence among boys in Indonesia. $\mathrm{Ng}$ et al. ${ }^{6}$ reported that some Indonesian boys believe that they have physical resilience to the harms of smoking and that it minimises its risk, while associating smoking with masculine characteristics and believing that engaging in smoking depicts them as being different

\section{from girls.}

Adolescence has specific characteristics related to psychological development, ability to process information, and tendency for risk minimisation. Adolescents, in general, have a tendency to feel invincible ${ }^{15}$ and according to Moutsiana et al. ${ }^{16}$, this age group has an imbalanced capacity to process desirable and undesirable information, with lower ability to process the undesirable information (i.e. the effect presented was worse than their estimation). Therefore, adolescents might have a good understanding of the health hazards of smoking, but at the same time they may also believe that smoking can be safe for a short period. Holding such opposing beliefs is similar to the concept of risk minimisation, which Helweg-Larsen et al. ${ }^{17}$ used to denote acknowledgement that smoking is dangerous, but at the same time, its risks can be underestimated. For boys and young men, physical risk is naturalised, promoted, and celebrated.

A recent WHO European region report acknowledged that tobacco use is strongly determined by gender norms with men perceiving their bodies as agents of gendered social practice, which they demonstrate through risk taking and risk minimisation ${ }^{18}$. In the context of the masculinised smoking issue in Indonesia, a lower ability to engage with undesirable information is a further disadvantage to boys and is reflected in the smoking problem among them. Adolescence is also a crucial stage when individual identities (psychological, social, and gender) and beliefs are developed, and behaviours adopted ${ }^{19}$ including the advantages and disadvantages related to smoking. Erikson's psychosocial developmental stage of adolescence named 'identity versus role confusion' frames these identities, beliefs and behaviours as taking place through role experimentation and exploration against their social circle, especially their peers and family ${ }^{19}$. Unfortunately, some beliefs favour smoking where it is construed as conferring social benefits.

Beliefs of social benefits have been reported as individual variables from other countries (such as Myanmar and Thailand) using GYTS data, such as smoking to feel more comfortable ${ }^{20}$, to have more friends ${ }^{20}$, and to be more attractive ${ }^{21}$, and these measures were reported to be higher among boys than girls. However, only the aspect of 
smoking to feel more comfortable was published in the Indonesian survey ${ }^{8}$. In different studies, such measures have been discussed under conceptual terms of functional beliefs ${ }^{22}$ or cognitive attributions ${ }^{23}$ along with other variables such as social image, social belonging, and smoking enjoyment. Such conceptual terms are highly relevant to the examination of smoking issues among boys as these intersect with perceived social benefits that are correlated differently to different points on smoking trajectories ${ }^{22}$. For example, social image and social belonging were found to be more highly correlated at earlier stages ${ }^{23}$ while smoking enjoyment was negatively related to quitting attempts ${ }^{22}$.

Monitoring tobacco use among Indonesian adolescents has been implemented using the GYTS since $2006^{24}$. The current study selected items from the GYTS to examine patterns of smoking behaviour in relation to masculinity to ensure the problem of smoking among Indonesian boys becomes more visible within the context of health promotion. In the current study, measures of smoking-related beliefs from the Indonesian GYTS 2014 are used to capture social and gendered belief concepts related to smoking and to explore how these are associated with several smoking behaviour outcomes among boys aged $12-16$ years.

\section{METHODS}

\section{Study overview}

The current study used data collected from the Indonesian GYTS, a nationally representative schoolbased survey. The GYTS collects data on tobacco consumption and initiation of tobacco use of students aged 12-16 years using a standard protocol across countries. The GYTS employed a two-stage cluster sample design of students in grades 7, 8 and 9. In the first stage, schools were selected with a probability proportional to school enrolment size, the school response rate was $100 \%$. The second sampling stage consisted of systematic equal probability sampling with a random start of classes from each school selected from the first stage, the overall class response rate was $98.1 \%$, and the student response rate was $91 \%$. The GYTS uses self-report questionnaires, including items on the frequency of cigarette and other tobacco use, exposure to secondhand smoke, access and availability of tobacco, whether they are taught about the dangers of tobacco use in class, awareness of media and advertising, smoking cessation, knowledge, and attitudes. Detailed information on the methodology of the survey has been reported in the Indonesian GYTS 2014 report $^{8}$. In the current study, data collected for 2100 Indonesian boys were included in the analysis after excluding 629 individuals due to missing data in any included variables. Ethical clearance of the original study was granted from Ethical Committee of National Institute of Health Research and Development, Indonesia (No LB.02.01/5.2/KE.424/2013).

\section{Measures}

Smoking behaviour outcomes as the main dependent variables of this study were: 1) current smoking, 2) smoking cessation (3 variables), and 3) susceptibility to future tobacco use (aggregate of two questions). Current smoking was defined as smoking cigarettes on 1 or more days in the last 30 days. Measures of smoking cessation were derived from current smokers and comprised three variables: intended to quit smoking, attempted to quit smoking in the past 12 months, and received help or advice to stop smoking from a program or professional, a friend and/or a family member, or from all three options. Susceptibility to future tobacco use was defined as non-smokers who might accept an offer from their best friends to smoke or to use tobacco during the next 12 months.

The main independent variables included in the study were two dimensions of smoking-related beliefs derived from Principal Component Analysis (PCA - further details below) of responses to eight questions. These questions were beliefs that: smoking is harmful, smoking is safe if it only lasts one or two years, secondhand smoke is harmful, enjoy smoking, smoking helps to feel comfortable at social events, smoking helps to have more friends, smoking to be more attractive, and smoking is difficult to quit. Detailed information on dependent and independent variables is presented in Table 1 . Potential covariates included in the study were: age, smoking dependence, father smokes, closest friends smoke, and students in the same grade smoke. Age was in discreet 1-year units from 12 to 16 years. Smoking dependence was measured by responses to smoke or feel like smoking tobacco first thing in the morning or start to feel a strong desire to smoke again within one full day after smoking. Mothers' 
Table 1. List of dichotomised dependent and independent variables

Variables Original questions

Based on response (Coded 1$)^{\text {a }}$

\section{Dependent variables}

Current smoking

Intention to quit

Attempted to quit

Received help

Susceptibility to future tobacco use

\section{Independent variables}

Smoking to feel more comfortable at social events

Enjoy smoking

Smoking to have more friends

Smoking to be more attractive

Smoking is harmful

Safe to smoke for one or two years

Secondhand smoke is harmful

Difficult to quit smoking

Smoking dependence

Father smokes

Closest friends smoke

Students in the same grade smoke
During the past 30 days, on how many days did you smoke cigarettes?

Do you want to stop smoking now?

During the past 12 months, did you ever try to stop smoking?

Have you ever received help or advice to help you to stop Yes smoking?

1. If one of your best friends offered you a tobacco product, would you use it?

2. At any time during the next 12 months, do you think you will use any form of tobacco?

Do you think smoking tobacco helps people feel more comfortable or less comfortable at celebrations, parties, or in another social gathering?

Do you agree or disagree with the following: 'I think I might enjoy smoking a cigarette'

Do you think young people who smoke tobacco have more or less friends?

Do you think smoking tobacco makes young people look more or less attractive?

Do you think smoking tobacco is harmful to your health?

Do you think it is safe to smoke tobacco for only a year or two, as long as you quit after that?

Do you think the smoke from other people's tobacco smoking is harmful to you?

Once someone has started smoking tobacco, do you think it would be difficult for them to quit?

Do you ever smoke tobacco or feel like smoking tobacco first thing in the morning?

Do your parents smoke tobacco?

Do any of your closest friends smoke tobacco?

About how many students in your grade smoke tobacco?
1 or more days

Yes

Yes

'definitely yes' and/or 'probably yes' and/or 'probably not'

More comfortable

'agree' and/or 'strongly agree' and/or 'I currently smoke cigarettes'

'more friends'

'more attractive'

'probably yes' and/or 'definitely yes' 'probably yes' and/or 'definitely yes' 'probably yes' and/or 'definitely yes.'

'probably yes' and/or 'definitely yes.'

Ever smoke or feel like smoking tobacco first thing in the morning

'father only' and/or 'both'

'some of them' and/or 'most of them' and/or 'all of them'

'about half of them' and/or 'some of them' and/or 'none of them'

a Other responses were coded as 0 .

smoking was not included as a measure in the current study because of the very low prevalence of smoking among women in Indonesia ${ }^{1,25}$.

\section{Statistical analysis}

Frequency and percentage of smoking-related behaviours and beliefs were computed by age group. Chi-squared test was used to test for statistical difference by age using a p-value lower than 0.05 .
PCA was performed to reduce eight smoking-related beliefs variables to meaningful components with the maintenance of as much information as possible. Selection of components was based on eigenvalues with values $>1$ for each individual variable. Varimax rotation was used to find each variable's correlation to the components. Loadings above 0.3 were considered important to decide with which component a variable was most strongly associated. Two new variables with 
scores predicted from the PCA analysis were generated and used for the multivariate logistic regression.

Multivariate logistic regressions were performed to test the association between the two components of smoking-related beliefs and the dependent variables (current smoking, intention to quit, attempt to quit, received help to quit, and susceptibility to smoking), controlling for potential covariates. The goodness-of-fit for models was tested using the Hosmer-Lemeshow test. Bootstrap syntax was employed to overcome issues of potential bias in participating schools, classes and students, and in students included in the analyses. All statistical procedures used STATA Version 12.

\section{RESULTS}

\section{Descriptive and bivariate relationships}

Prevalence of current smoking increased with the age of study participants (Table 2 ); with $23.9 \%$ of participants smoking at age 12 years followed by a steep increase until the ages of 15 and 16 years, when $60 \%$ of participants smoked.

Table 2 shows the frequency and percentage of smoking-related behaviours and beliefs of study participants. The majority of boys aged 16 years who were current smokers received help to quit smoking $(95.2 \%)$. An increased proportion of smoking dependence was observed among older boys (ages of 15 and 16 years). The susceptibility to smoking increased by age, with the highest percentage of smokers among boys aged 16 years.

In relation to smoking beliefs, it is important to note that beliefs regarding the harms of smoking and secondhand smoke were relatively the same across ages 12 to 16 years. In contrast, beliefs on smoking to help feel comfortable at social events, enjoying smoking, smoking to have more friends, smoking to be more attractive, and that it is safe to smoke for one or two

Table 2. Frequency and percentage of smoking related behaviours and beliefs by age

\begin{tabular}{|c|c|c|c|c|c|c|}
\hline & \multicolumn{3}{|l|}{ Total } & \multicolumn{3}{|l|}{ Age (year) } \\
\hline & n & $\begin{array}{l}\leq 12 \\
\text { II }(\%)\end{array}$ & $\begin{array}{c}13 \\
n(\%)\end{array}$ & $\begin{array}{c}11 \\
n(\%)\end{array}$ & $\begin{array}{c}15 \\
11(\%)\end{array}$ & 16 \\
\hline \multicolumn{7}{|l|}{ Dependent variables } \\
\hline Current smoking & 2100 & $113(23.9)$ & $234(29.5)$ & $230(41.8)$ & $129(60)$ & $42(60)$ \\
\hline Desire to quit & 748 & $80(70.8)$ & $172(73.5)$ & $169(73.5)$ & $88(68.2)$ & $31(73.8)$ \\
\hline Attempt to quit* & 748 & $73(64.6)$ & $166(70.9)$ & $169(73.5)$ & $76(58.9)$ & $29(69.0)$ \\
\hline Received help to quit & 748 & $100(88.5)$ & $212(90.6)$ & $199(86.5)$ & $117(90.7)$ & $40(95.2)$ \\
\hline Susceptibility to future smoke & 1218 & $48(14.2)$ & $80(15.8)$ & $43(15.9)$ & $10(13)$ & $6(24)$ \\
\hline \multicolumn{7}{|l|}{ Independent variables } \\
\hline Smoking dependency* & 748 & $16(14.2)$ & $50(21.4)$ & $50(21.7)$ & $40(31.0)$ & $13(30.9)$ \\
\hline \multicolumn{7}{|l|}{ Beliefs of smoking } \\
\hline $\begin{array}{l}\text { Smoking to feel more comfortable at } \\
\text { social events* }\end{array}$ & 2100 & $45(9.5)$ & $83(10.5)$ & $65(11.8)$ & $39(18.1)$ & $10(14.3)$ \\
\hline Enjoy smoking* & 2100 & $75(15.9)$ & $119(15)$ & $138(25.1)$ & $74(34.4)$ & $23(32.9)$ \\
\hline Smoking to have more friends* & 2100 & $98(20.8)$ & $177(22.3)$ & $156(28.4)$ & $77(35.8)$ & $23(32.9)$ \\
\hline Smoking to be more attractive ${ }^{*}$ & 2100 & $28(5.93)$ & $61(7.7)$ & $53(9.6)$ & $32(14.9)$ & $8(11.4)$ \\
\hline Smoking is harmful & 2100 & $425(90)$ & 727 (91.7) & $509(92.5)$ & $198(92.1)$ & $63(90)$ \\
\hline Safe to smoke for one or two years* & 2100 & $137(29)$ & $264(33.3)$ & $190(34.5)$ & $100(46.5)$ & $37(52.9)$ \\
\hline Secondhand smoke is harmful & 2100 & $452(95.8)$ & $746(94.1)$ & $516(93.8)$ & $199(92.6)$ & $66(94.3)$ \\
\hline Difficult to quit smoking & 2100 & $368(78)$ & $603(76)$ & $415(75.4)$ & $160(74.4)$ & $53(75.7)$ \\
\hline \multicolumn{7}{|l|}{ Social circles } \\
\hline Father smokes* & 2100 & $274(58)$ & $499(62.9)$ & $379(68.9)$ & $150(69.8)$ & $49(70)$ \\
\hline Closest friends smoke* & 2100 & $263(55.7)$ & $533(67.2)$ & $440(80)$ & $182(84.6)$ & $63(90)$ \\
\hline Students in same grade smoke* & 2100 & $321(68)$ & $601(75.8)$ & $464(84.4)$ & $191(88.8)$ & $63(90)$ \\
\hline
\end{tabular}

${ }^{*}$ Chi-squared test of relationships between smoking-related behaviours and age $p<0.05$. 
years, significantly increased with age. The proportion of those whose father smoked, closest friends smoked, and students in the same grade smoked, also significantly increased with participants age.

\section{PCA results}

Two components were derived from the eight items entered into the PCA, each component comprising four items. The first component included variables describing smoking to make one feel more comfortable at social events, enjoy smoking, have more friends, and make oneself more attractive (contributed $23.5 \%$ of variance). This component was labelled as perceived

Table 3. Principle Components Analysis of smoking related belief items-varimax rotation

\begin{tabular}{|c|c|c|c|}
\hline \multicolumn{2}{|r|}{$\begin{array}{l}\text { Items measuring smoking } \\
\text { related beliefs }\end{array}$} & \multirow{2}{*}{$\begin{array}{c}\text { Perceived } \\
\text { social } \\
\text { benefits } \\
\text { Component } \\
1 \\
0.33\end{array}$} & \multirow{2}{*}{$\begin{array}{l}\text { Perceived } \\
\text { Inarm } \\
\text { Componem } \\
2 \\
0.03\end{array}$} \\
\hline 1 & $\begin{array}{l}\text { Smoking to feel more } \\
\text { comfortable at social events }\end{array}$ & & \\
\hline 2 & Enjoy smoking & 0.38 & 0.03 \\
\hline 3 & Smoking to have more friends & 0.33 & -0.04 \\
\hline 4 & Smoking to be more attractive & 0.37 & -0.04 \\
\hline 5 & Smoking is harmful & -0.01 & 0.49 \\
\hline 6 & $\begin{array}{l}\text { Safe to smoke for one or two } \\
\text { years }\end{array}$ & 0.28 & 0.31 \\
\hline 7 & Secondhand smoke is harmful & -0.01 & 0.43 \\
\hline 8 & Difficult to quit smoking & 0.07 & 0.45 \\
\hline & Eigenvalue & 1.75 & 1.38 \\
\hline & Variance explained (\%) & 23.5 & 15.7 \\
\hline
\end{tabular}

social benefits of smoking. The second component, labelled as perceived harms, included variables describing smoking as harmful, smoking is safe if it only lasts one or two years, secondhand smoke is harmful, and it is difficult to quit smoking (contributed $15.7 \%$ of the variance). Loadings for each variable and the components are shown in Table 3.

\section{Associations between smoking and cessation behaviour and perceived harms and benefits of smoking}

In analyses adjusting for potential confounders, the variable 'perceived social benefits' was positively associated with being a current smoker ( $\mathrm{OR}=2.5 ; 95 \%$ CI: 2.2-2.8) while 'perceived harms' was negatively associated with being a current smoker $(\mathrm{OR}=0.9$; 95\% CI: 0.8-1.0). In terms of smoking cessation measures, an increase in the perceived social benefits score was negatively associated with respondents' intention to quit smoking ( $\mathrm{OR}=0.9$; 95\% CI: 0.7-1.0). Conversely, a higher score for perceived harms was positively associated with respondents' intention to quit smoking ( $\mathrm{OR}=1.2 ; 95 \% \mathrm{CI}$ : 1.1-1.5). Increases in perceived harms were also positively associated with respondents' attempts to stop smoking $(\mathrm{OR}=1.3$; 95\% CI: 1.1-1.5). Increasing scores on perceived social benefits were positively associated with being susceptible to future tobacco use $(\mathrm{OR}=1.6 ; 95 \% \mathrm{CI}$ : $1.3-2.0)$, especially for boys aged 12 and 13 years. Meanwhile, a higher score for perceived harms was negatively associated with respondents' susceptibility to future tobacco use $(\mathrm{OR}=0.8 ; 95 \%$ CI: 0.7-0.9). Detail results are presented in Table 4 .

Table 4. Multivariate logistic regression of smoking-related beliefs and smoking outcomes

\begin{tabular}{|c|c|c|c|c|c|}
\hline \multirow[t]{3}{*}{ Variables } & \multirow{2}{*}{$\begin{array}{l}\text { Current } \\
\text { smokingt }\end{array}$} & \multicolumn{3}{|c|}{ Smoking cessation measures } & \multirow{2}{*}{$\begin{array}{l}\text { Susceptibility } \\
\text { to future } \\
\text { tobacco use }\end{array}$} \\
\hline & & Desire to quit ${ }^{2}$ & $\begin{array}{l}\text { Atempted to } \\
\text { quit }^{3}\end{array}$ & $\begin{array}{l}\text { Received help } \\
\text { to quit' }\end{array}$ & \\
\hline & $\begin{array}{c}\text { OR } \\
\left(95^{\circ} \% \mathrm{CI}\right)\end{array}$ & $\begin{array}{c}\text { OR } \\
(95 \% \mathrm{CI})\end{array}$ & $\begin{array}{c}\text { OR } \\
\left(95^{\circ} \% \mathrm{CI}\right)\end{array}$ & $\begin{array}{c}\text { OR } \\
\left(95^{\circ} \% \mathrm{CI}\right)\end{array}$ & $\begin{array}{c}\text { OR } \\
(95 \% \mathrm{CI})\end{array}$ \\
\hline \multicolumn{6}{|l|}{ Smoking-related beliefs } \\
\hline Perceived social benefit & $\begin{array}{c}2.46^{*} \\
(2.17-2.79)\end{array}$ & $\begin{array}{c}0.85^{*} \\
(0.72-0.99)\end{array}$ & $\begin{array}{c}0.99 \\
(0.87-1.14)\end{array}$ & $\begin{array}{c}1.03 \\
(0.80-1.32)\end{array}$ & $\begin{array}{c}1.58^{*} \\
(1.27-1.97)\end{array}$ \\
\hline Perceived harm & $\begin{array}{c}0.88^{*} \\
(0.78-0.99)\end{array}$ & $\begin{array}{c}1.25^{*} \\
(1.07-1.47)\end{array}$ & $\begin{array}{c}1.29^{*} \\
(1.12-1.48)\end{array}$ & $\begin{array}{c}1.15 \\
(0.96-1.39)\end{array}$ & $\begin{array}{c}0.79^{*} \\
(0.66-0.94)\end{array}$ \\
\hline
\end{tabular}


Table 4. Continued

\begin{tabular}{|c|c|c|c|c|c|}
\hline \multirow[t]{3}{*}{ Variables } & \multirow{3}{*}{$\begin{array}{c}\text { Current } \\
\text { smoking } \\
\\
\text { OR } \\
(95 \% \mathrm{CI})\end{array}$} & \multicolumn{3}{|c|}{ Smoking cessation measures } & \multirow{2}{*}{$\begin{array}{c}\text { Susceptibility } \\
\text { to future } \\
\text { tobacco use }\end{array}$} \\
\hline & & Desire to quit ${ }^{2}$ & $\begin{array}{l}\text { Attempted to } \\
\text { quit }{ }^{3}\end{array}$ & $\begin{array}{l}\text { Received help } \\
\text { to quit' }\end{array}$ & \\
\hline & & $\begin{array}{c}\text { OR } \\
(95 \% \text { CI })\end{array}$ & $\begin{array}{c}\text { OR } \\
\left(95^{\circ} \% \mathrm{CI}\right)\end{array}$ & $\begin{array}{c}\text { OR } \\
(95 \% \text { CI })\end{array}$ & $\begin{array}{c}\text { OR } \\
\left(95^{\circ} \% \mathrm{CI}\right)\end{array}$ \\
\hline \multicolumn{6}{|l|}{ Age (years) (Ref. 12) } \\
\hline 13 & $\begin{array}{c}1.11 \\
(0.81-1.51)\end{array}$ & $\begin{array}{c}1.16 \\
(0.70-1.91)\end{array}$ & $\begin{array}{c}1.33 \\
(0.84-2.10)\end{array}$ & $\begin{array}{c}1.21 \\
(0.59-2.49)\end{array}$ & $\begin{array}{c}1.08 \\
(0.78-1.49)\end{array}$ \\
\hline 14 & $\begin{array}{c}1.44^{*} \\
(1.08-1.91)\end{array}$ & $\begin{array}{c}1.14 \\
(0.67-1.94)\end{array}$ & $\begin{array}{c}1.43 \\
(0.90-2.28)\end{array}$ & $\begin{array}{c}0.78 \\
(0.41-1.46)\end{array}$ & $\begin{array}{c}0.96 \\
(0.66-1.39)\end{array}$ \\
\hline 15 & $\begin{array}{c}2.64^{*} \\
(1.94-3.59)\end{array}$ & $\begin{array}{c}0.95 \\
(0.57-1.57)\end{array}$ & $\begin{array}{c}0.74 \\
(0.44-1.26)\end{array}$ & $\begin{array}{c}1.19 \\
(0.59-2.39)\end{array}$ & $\begin{array}{c}0.77 \\
(0.32-1.81)\end{array}$ \\
\hline 16 & $\begin{array}{c}2.45^{*} \\
(1.37-4.39)\end{array}$ & $\begin{array}{c}1.11 \\
(0.49-2.48)\end{array}$ & $\begin{array}{c}1.05 \\
(0.46-2.35)\end{array}$ & $\begin{array}{c}2.48 \\
(0.75-8.23)\end{array}$ & $\begin{array}{c}1.29 \\
(0.52-3.19)\end{array}$ \\
\hline Smoking dependency (Ref. No) & & $\begin{array}{c}0.73 \\
(0.50-1.07)\end{array}$ & $\begin{array}{c}0.90 \\
(0.61-1.31)\end{array}$ & $\begin{array}{c}1.13 \\
(0.55-2.29)\end{array}$ & \\
\hline Father smokes (Ref. No) & $\begin{array}{c}1.71^{*} \\
(1.35-2.17)\end{array}$ & $\begin{array}{c}0.79 \\
(0.54-1.18)\end{array}$ & $\begin{array}{c}0.65^{*} \\
(0.46-0.93)\end{array}$ & $\begin{array}{c}1.93^{*} \\
(1.12-3.32)\end{array}$ & $\begin{array}{c}1.63^{*} \\
(1.11-2.39)\end{array}$ \\
\hline Closest friends smoke (Ref. No) & $\begin{array}{c}6.18^{*} \\
(4.32-8.86)\end{array}$ & $\begin{array}{c}0.93 \\
(0.42-2.08)\end{array}$ & $\begin{array}{c}0.76 \\
(0.35-1.63)\end{array}$ & $\begin{array}{c}1.38 \\
(0.31-6.12)\end{array}$ & $\begin{array}{c}1.11 \\
(0.72-1.69)\end{array}$ \\
\hline Students in same grade smoke (Ref. No) & $\begin{array}{c}1.65^{*} \\
(1.09-2.49)\end{array}$ & $\begin{array}{c}1.67 \\
(0.74-3.77)\end{array}$ & $\begin{array}{c}1.94 \\
(0.88-4.25)\end{array}$ & $\begin{array}{c}1.20 \\
(0.21-6.81)\end{array}$ & $\begin{array}{c}1.74^{*} \\
(1.11-2.73)\end{array}$ \\
\hline \multicolumn{6}{|l|}{ Model parameters } \\
\hline Number of observations & 2100 & 748 & 748 & 748 & 1218 \\
\hline \multicolumn{6}{|l|}{ Hosmer-Lemeshow } \\
\hline$p$-value & 0.74 & 0.62 & 0.23 & 0.16 & 0.54 \\
\hline Pseudo $\mathrm{R}^{2}$ & 0.26 & 0.03 & 0.03 & 0.03 & 0.06 \\
\hline
\end{tabular}

Perceived social benefit (low score $=$ low benefit). Perceived benefit (low score $=$ greater harm perceived). ${ }^{*} \mathrm{p}<0.05$

Age was significantly associated with current smoking status. No such associated was found between age and smoking cessation or susceptibility to future tobacco use. Having a father that smokes was positively associated with being a current smoker (OR=1.7; 95\% CI: $1.3-2.2)$, receiving some help to do so $(\mathrm{OR}=1.9 ; 95 \% \mathrm{CI}: 1.1-3.3)$ and being susceptible to future smoking $(\mathrm{OR}=1.6 ; 95 \% \mathrm{CI}$ : 1.1-2.5). In contrast, having a father who smokes was also negatively associated with attempting to quit $(\mathrm{OR}=0.6 ; 95 \%$ CI: $0.5-0.9)$. Having best friends who smoked was positively associated with being a current smoker (OR=6.2; 95\% CI: 4.3-8.9). Having peers from the same grade who smoked had significantly higher odds of engaging in current smoking ( $\mathrm{OR}=1.6 ; 95 \% \mathrm{CI}: 1.1-2.5)$ or having susceptibility to future smoking $(\mathrm{OR}=1.7 ; 95 \% \mathrm{CI}$ : $1.1-2.7)$.

\section{DISCUSSION}

This study focused on smoking behaviour among Indonesian boys aged 12-16 years. This is a lifestage during which prevalence of smoking increases and therefore poses the most pressing public health concern. Along with this high prevalence, there is increasing concern about social-related factors that have been shown to be crucial for framing adolescents' behaviours regarding smoking, as presented in a

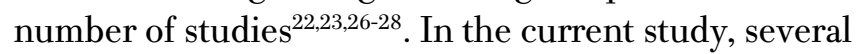
items measuring such factors were included. PCA was 
used to capture concepts of smoking-related beliefs among boys from these measures. Two components were identified, representing men's tendency to perform risk minimisation of the hazards of tobacco use during adolescence. The theoretical framework of HIMM ${ }^{13}$ explained how those two components associated with smoking in opposite ways reflected boys' masculine tendency of risk taking and risk minimisation that intersects with age. The main findings were that year-by-year during adolescence, smoking among Indonesian boys increased rapidly and was positively associated with beliefs regarding perceived social benefits and negatively with beliefs regarding perceived harms. These findings are discussed in the context of boys' cultural, social, and gendered environment in Indonesia.

\section{Components of smoking-related beliefs}

Given the importance of social aspects for determining adolescent boys' smoking behaviours, in the current study eight GYTS individual items measuring smoking-related beliefs were categorised into two components, perceived social benefits and perceived harms, based on PCA analysis. The components explained $39.2 \%$ of variance of smoking behaviours, forming reasonable measures of these two belief components. However, they are likely to have missed some essential aspects that will require further exploration to develop them. The higher contribution of variance to the construct 'perceived social benefits' (23.5\%) compared to 'perceived harms' (15.7\%) illustrates the first component could explain more of beliefs compared to that of perceived harms and therefore it is important to focus on social-related beliefs.

Measures of social-related factors and beliefs have been acknowledged and employed in several smoking studies focusing on youth elsewhere ${ }^{22,23,26-28}$ as this aspect is important for adolescents' development. Erikson's developmental theory positions adolescents at a stage where they evaluate their beliefs, values, and actions through experimentation and exploration against their social circle, especially their peers and family. Therefore, it is important to examine what Indonesian boys are learning about social benefits of smoking at this age. In their current form, this component has shown strong associations to smoking behaviours and revealed important relationships with these behaviours. The perceived social benefits described in this study have similarity with the concept of functional beliefs by Yong et al. ${ }^{22}$ along with other measures such as use for enjoyment and stress management. Such beliefs, which are perceived as reasons for smoking, were defined as cognitive attributions by Guo et al. ${ }^{23}$. Both studies found that these beliefs were more prevalent among boys ${ }^{23}$, indicating the importance of studying perceptions of social benefits of smoking behaviours among this group.

\section{Relationships between smoking-related beliefs, smoking behaviours}

At an international level, Indonesia represents a special context in terms of smoking as it has been unable to report any reduction in smoking prevalence among boys over time ${ }^{1}$. The Global Burden of Disease (GBD) study showed that Indonesia is the only country among those in the top ten with the highest smoking prevalence in the world that still has a positive growth of prevalence among boys (age 15-19 years $)^{1}$. This increase in prevalence remains up to the age of 55 years $^{29}$. The years during adolescence are critical given that smoking initiation among men in many countries occurs mostly during this period ${ }^{3}$. This study confirmed the GBD trend in Indonesia that current smoking during adolescence among boys increased sharply year-by-year, with prevalence among boys almost tripling between ages 12 and 16 years. Therefore, future studies and interventions are urgently required to tackle the smoking problem in this group.

Despite the importance of measuring smoking prevalence, having measures of smoking-related beliefs and percentage of smokers among those in the boys' social circles, in relation to their developmental stage, are crucial for understanding smoking behaviours. It is important to note that the current study only included four measures of perceived harm of smoking (beliefs of smoking and secondhand smoke are harmful, safe to smoke for one or two years, and difficult to quit smoking) all of which remained stable across each age except for 'safe to smoke for one or two years' which increased with age. The question on 'safe to smoke for one or two years' might reflect low risk perception of 
smoking in the Indonesia setting, which is in line with a study conducted in the Virginia population ${ }^{30}$. They found that 'safe to smoke for one or two years' was positively associated with smoking due to belief that it is easy to quit smoking as well as low risk perception of smoking. However, the need to do further investigation through more specific questions on the harmful aspect of smoking for a short period is recommended. The response on the question 'safe to smoke for one or two years' may differ across different cultural contexts.

The four measures of beliefs that favour smoking (smoking to feel more comfortable at social event, enjoy smoking, smoking to have friends, and smoking to be more attractive) and measures of smokers in the boys' social circles, steadily increased year-by-year. When the measures were combined to derive the components 'perceived social benefits' and 'perceived harms' and adjusted for other covariates, these were consistently but conversely associated with smoking behaviour outcomes. For example, perceived social benefits were associated with increasing susceptibility to future tobacco use but perceived harms were associated with lower use.

It is not surprising that these two components showed associations in opposite directions since they represent opposing belief constructs. These opposing responses to social benefit and perceived harms are consistent with the Helweg-Larsen et al. ${ }^{17}$ concept of risk minimisation. Their study, based in the United States and Denmark, defined risk minimisation as being skeptical about warning messages related to smoking, thinking that one is exempt from the health hazards of tobacco, viewing smoking as just one of many dangerous things that could happen, and believing any harm will occur at some time in the future. Similar findings from a qualitative study of adolescents in Indonesia by $\mathrm{Ng}$ et al. ${ }^{6}$ found that some Indonesian boys were not afraid of health hazards caused by tobacco. Subramaniam et al. ${ }^{31}$ found in Singapore, which neighbours Indonesia, that some adolescents believed smoking has adverse health outcomes but at the same time minimised the risk of adverse health outcomes. Even though studies have indicated that lack of knowledge about the harm of tobacco is an important factor for smoking among adolescents ${ }^{32}$, findings of this study indicate a further need to address issues of the perceived social benefits of smoking. The reduced desire to quit and increased susceptibility to smoking in the future with higher perceived social benefits, further highlight its importance. Such perceived social benefits of smoking need to be monitored and countered by future studies and interventions.

\section{Social benefit beliefs and the social circle}

In the current study, smoking within the boys' closest social circles was associated with their current smoking and their susceptibility to smoking. Several studies have revealed such correlations between boys' smoking status and that of their parents ${ }^{31,33}$ and/or friends ${ }^{31,33,34}$. It is important to note that the father's smoking status was positively associated with the boys' smoking status for several outcomes, including current smoking, received help to quit, and susceptibility to future tobacco use. Such findings might be explained by the boys' gender identity development. Several studies had reported the prevalence of smoking among adult women to be very low ${ }^{1,29}$ (making prevalence of smoking among mothers an unsuitable measure for inclusion in the current study), contrasting with the high prevalence of fathers' smoking. In this context, following gender identity formation theory, Indonesian boys might perceive smoking as a means of differentiating their gender identity from that of their mothers' and to more closely reflect their fathers' ${ }^{35}$.

However, it is uncertain whether the agerelated increase in beliefs favourable to smoking occurs actively to conform to boys' social circles or passively due to social pressure from their social circles. It may be an interplay of both, given the boys' developmental stage as this is an age during which they often experiment with their values against those of their social circle ${ }^{19}$. The $\mathrm{Ng}$ et al. ${ }^{6}$ qualitative study among Indonesian adolescent boys revealed similar findings regarding conformity to peers in their social circles. The relationship of smoking among boys and that of their social circle is particularly problematic for boys in the context of gender identity formation given the current high prevalence of smoking among boys and adult men in Indonesia and the representation of masculinised characteristics in association with smoking in tobacco industry advertising $^{7,8}$. 


\section{Recommendations for future research and practice}

Given the high and rapid increase of smoking prevalence among boys in Indonesia, we recommend that future tobacco control research and practice should specifically target boys and factors related to the perceived social benefits of smoking and other beliefs favouring smoking. Such research will fill a critical gap in our understanding of the association between smoking, social benefits and masculinity among boys ${ }^{12}$ and will also inform the development of interventions that target this high risk and vulnerable group.

Based on several recent systematic reviews ${ }^{27,28}$, it is clear that there is a paucity of intervention studies that address smoking prevention and cessation among boys. Current interventions developed for adolescents, in general, have incorporated aspects of social skills such as refusal skills, selfcontrol, communication, and self-improvement ${ }^{27,28}$. Interventions to date fail to take into consideration boys' characteristics and beliefs. Social skills need to be contextualised within the developing gender identities of adolescent boys and monitored using measures that can capture social benefit beliefs, social and gender influences and the risk minimisation behaviours within large scale surveys such as the GYTS.

Positive messages of the harms of smoking and benefits of giving up smoking, particularly to support a social life, are also warranted. Such positive messages and campaigns may also help overcome the negation or disregard of warnings and the influences that encourage engagement in risky behaviours; such interventions are urgently required for boys at the adolescent stage of their development ${ }^{14}$.

\section{Strengths and limitations}

The main strength of this study is that the dataset used is from a nationally representative survey, designed specifically for examining smoking behaviour among adolescents. PCA employed in this study enabled the inclusion of two components capturing dimensions of social benefits and harms of smoking in order to increase understanding of their relationship to smoking behaviours.

Details of the limitations of the GYTS design have been described elsewhere ${ }^{20,33}$, in summary, the main limitations relate to the data being collected from school adolescents, therefore, findings cannot be generalised to all adolescents. Also of note is that the original study was cross-sectional in design, therefore causality cannot be determined from the data.

Only very limited aspects of gender are captured from this survey, which is confined to whether the respondent is male or female. Hence, it is difficult to examine aspects of masculinity in relation to smoking among boys. This current situation lends support to Kimmel's argument that because men's characteristics have been regarded as the norm within most cultures, their experience or problems tend to be overlooked ${ }^{36}$. Given the context of smoking behaviours in Indonesia, the focus of gender issues in smoking needs to proportionally emphasise the burden among adolescent boys. Therefore, the continuing high smoking prevalence among boys in Indonesia necessitates a specific focus on this group in the context of cultural, social and gender influences on their behaviours.

As the GYTS survey did not specifically explore smoking-related beliefs some known aspects of smoking-related beliefs such as those reported by Yong and Borland ${ }^{22}$, could not be included in the current study. Missing aspects such as smoking to help concentration and smoking as a life enhancement (smoking is an important part of life), and smoking as a means of stress management could be important measures worth consideration for future inclusion that could add value to the next GYTS survey. The measures collected for the survey enabled extraction of components representing both perceived social benefits and perceived harms of smoking. Furthermore, beliefs related to boys' masculine identity could also be a valuable addition in future surveys, such as smoking to make them feel like a man or smoking to differ from girls/women. Despite limitations on the extent of measures, this study provides a starting point for further research in smoking control among boys, which is of particular concern in Indonesia.

\section{CONCLUSIONS}

Indonesian school boys, aged 12-16 years, experience a rapid increase in smoking outcomes and a corresponding increase in smoking among their social circle. The high sustained percentage 
reporting they believe that smoking is harmful but increase in those reporting perceived social benefits during this period is similar to the concept of risk minimisation, which is closely related to the masculine tendency to undermine health hazards of tobacco. Therefore, it is important that future work addresses these highly gender-related issues within Indonesia, including the monitoring of smoking prevalence and perceived social benefits of smoking as well as developing interventions that target this at-risk group (i.e. Indonesian boys aged 12-16 years).

\section{REFERENCES}

1. Reitsma M, Fullman N, Ng M, et al. Smoking prevalence and attributable disease burden in 195 countries and territories, 1990-2015: a systematic analysis from the Global Burden of Disease Study 2015. Lancet. 2017;389(10082):18851906. doi:10.1016/S0140-6736(17)30819-X

2. Global Youth Tobacco Survey Collaborating Group. Differences in worldwide tobacco use by gender: Findings from the global youth tobacco survey. J Sch health. 2003;73(6):207-215. doi:10.1111/j.1746-1561.2003.tb06562.x

3. Talip T, Murang Z, Kifli N, Naing L. Systematic Review of Smoking Initiation among Asian Adolescents, 20052015: Utilizing the Frameworks of Triadic Influence and Planned Behavior. Asian Pac J Cancer Prev. 2016;17(7):3341-3355.

4. Institute for Health Metrics and Evaluation. Indonesia male daily smoking prevalence(\%). http://www. healthdata.org/smoking-tobacco. Published 2017. Accessed April 11, 2019.

5. Roberts BB. The "Marlboro Men" of the Early Seventeenth Century: Masculine Role Models for Dutch Youths in the Golden Age? Men and Masculinities. 2006;9(1):76-94. doi:10.1177/1097184x05283483

6. $\mathrm{Ng} \mathrm{N}$, Weinehall L, Ohman A. 'If I don't smoke, I'm not a real man'--Indonesian teenage boys' views about smoking. Health Educ Res. 2007;22(6):794-804. doi:10.1093/her/cyl104

7. Nichter M, Padmawati S, Danardono M, Ng N, Prabandari Y, Mark N. Reading culture from tobacco advertisements in Indonesia. Tob Control. 2009;18(2):98-107. doi:10.1136/tc.2008.025809

8. World Health Organization, Regional Office for South-East Asia. Global Youth Tobacco Survey (GYTS): Indonesia report, 2014. New Delhi: World Health Organization, Regional Office for South-East Asia; 2015. https://apps. who.int/iris/bitstream/handle/10665/205148/B5209. pdf? sequence=1\&isAllowed=y. Accessed June 3, 2019.

9. Priyatna CC. The Invisible Cigarette: The Production of Smoking Culture and Identity in Indonesia. Melbourne, Australia: Monash University; 2013. https://monash. figshare.com/articles/The_invisible_cigarette_the_ production_of_smoking_culture_and_identity_in_ Indonesia/4701415. Accessed June 3, 2019.

10. Nilan P. Contemporary masculinities and young men in Indonesia. Indonesia and the Malay World. 2009;37(109):327-344. doi:10.1080/13639810903269318

11. Connell R. Masculinities. Cambridge, United Kingdom: Polity Press; 2005.

12. Kodriati N, Pursell L, Hayati EN. A scoping review of men, masculinities, and smoking behavior: The importance of settings. Glob Health Act. 2018;11(sup3):1589763. doi:10.1080/16549716.2019.1589763

13. Evans J, Frank B, Oliffe JL, Gregory D. Health, illness, men, and masculinities (HIMM): A theoretical framework for understanding men and their health. JMH. 2011;8(1):7-15. doi:10.1016/j.jomh.2010.09.227

14. Gough B, Robertson S. Men, Masculinities, and Health. London, United Kingdom: Palgrave Macmillan; 2010.

15. Wickman ME, Anderson NLR, Smith Greenberg C. The Adolescent Perception of Invincibility and Its Influence on Teen Acceptance of Health Promotion Strategies. J Pediatr Nurs. 2008;23(6):460-68. doi:10.1016/j.pedn.2008.02.003

16. Moutsiana C, Garret N, Clarke RC, Lotto RB, Blakemore SJ, Sharot T. Human development of the ability to learn from bad news. PNAS. 2013;110(41):16396-16401. doi:10.1073/pnas.1305631110

17. Helweg-Larsen M, Tobias MR, Cerban BM. Risk perception and moralization among smokers in the USA and Denmark: A qualitative approach. Br J Health Psychol. 2010;15(4):871-886. doi:10.1348/135910710X490415

18. World Health Organization. Strategy on the health and well-being of men in the WHO European Region. Rome, Italy: World Health Organization Regional Office for Europe; 2018. http://www.euro.who.int/_data/assets/ pdf_file/0010/394894/MHR_strategy_Eng_online. pdf?ua=1. Accessed June 3, 2019.

19. Hutchison ED. Essentials of human behavior: integrating person, environment, and the life course. Los Angeles, CA: Sage; 2013.

20. Chotbenjamaporn P, Haruhansapong V, Jumriangrit P, Pitayarangsarit S, Agarwal N, Garg R. Tobacco use among thai students: Results from the 2015 global youth tobacco survey. Indian J Public Health. 2017;61(5):4046. doi:10.4103/ijph.IJPH_234_17

21. Rudatsikira E, Muula AS, Siziya S, Mataya RH. Correlates of cigarette smoking among school-going adolescents in Thailand: findings from the Thai global youth tobacco survey 2005. Int Arch Med. 2008;1(1):8. doi:10.1186/1755-7682-1-8

22. Yong HH, Borland R. Functional beliefs about smoking and quitting activity among adult smokers in four countries: findings from the International Tobacco Control FourCountry Survey. Health Psychol. 2008;27(3S):S216-S23. doi:10.1037/0278-6133.27.3(suppl.).s216

23. Guo Q, Unger JB, Azen SP, MacKinnon DP, Johnson CA. 
Do cognitive attributions for smoking predict subsequent smoking development? Addict Behav. 2012;37(3):273279. doi:10.1016/j.addbeh.2011.11.002

24. World Health Organization. Report on Global Youth Tobacco Survey (GYTS), Indonesia, 2006. New Delhi, India: World Health Organization Regional Office; 2006. https://extranet.who.int/ncdsmicrodata/index.php/ catalog/263. Accessed June 3, 2019.

25. Ng M, Freeman MK, Fleming TD, et al. Smoking prevalence and cigarette consumption in 187 countries, 1980-2012. JAMA. 2014;311(2):183-192. doi:10.1001/jama.2013.284692

26. Kasim K, Al-Zalabani A, Abd El-Moneim ES, Abd ElMoneim S. Beliefs and attitudes of male and female adolescents and the risk of smoking behavior. J Postgrad Med. 2016;62(2):80-85. doi:10.4103/0022-3859.180546

27. Thomas RE, Lorenzetti Dl Fau - Spragins W, Spragins W. Systematic review of mentoring to prevent or reduce tobacco use by adolescents. Acad Pediatr. 2013;13(4):300307. doi:10.1016/j.acap.2013.03.008

28. Peirson L, Ali MU, Kenny M, Raina P, Sherifali D. Interventions for prevention and treatment of tobacco smoking in school-aged children and adolescents: a systematic review and meta-analysis. Prev Med. 2016(85):20-31. doi:10.1016/j.ypmed.2015.12.004

29. Amalia B, Cadogan SL, Prabandari YS, Filippidis FT. Socio-demographic inequalities in cigarette smoking in Indonesia, 2007 to 2014. Prev Med. 2019;123:27-33. doi:10.1016/j.ypmed.2019.02.025

30. Jones RM, Wiseman KP, Kharitonova M. Association between high school students' cigarette smoking, asthma and related beliefs: a population-based study. BMC Public Health. 2016;16(1):913. doi:10.1186/s12889-016-3579-7

31. Subramaniam M, Shahwan S, Fauziana R, et al. Perspectives on Smoking Initiation and Maintenance: A Qualitative Exploration among Singapore Youth. Int J Environ Res Public Health. 2015;12(8):8956-8970. doi:10.3390/ijerph120808956

32. Bertani AL, Garcia T, Erico T, Godoy I. Tobacco in adolescence: Importance to knowledge the health hazards and preventive measures. J Addict Res Ther. 2015;6(1):1000218. doi:10.4172/2155-6105.1000218

33. Ribeiro Sarmento D, Yehadji D. An analysis of global youth tobacco survey for developing a comprehensive national smoking policy in Timor-Leste. BMC Public Health. 2016;16(1):65. doi:10.1186/s12889-016-2742-5

34. French DC, Purwono U, Rodkin P. Indonesian Muslim Adolescents' Use of Tobacco and Alcohol: Associations With Use by Friends and Network Affiliates. Merrill-Palmer Quarterly. 2014;60(4):385-402. doi:10.13110/merrpalmquar1982.60.4.0385

35. World Health Organization. What about boys? A literature review on the health and development of adolescent boys. Geneva, Switzerland: World Health Organization; 2000. https://apps.who.int/
iris/bitstream/handle/10665/66487/WHO_FCH_ CAH_00.7.pdf?sequence=1. Accessed June 3, 2019.

36. Kimmel MS. The gendered society. 4th ed. Oxford, United Kingdom: Oxford University Press; 2011.

\section{ACKNOWLEDGEMENTS}

We are grateful to Nawi $\mathrm{Ng}$ (Umea and Gothenburg University) for the opportunity to work closely with him. We thank Endang Indriasih, Principal Investigator of GYTS Indonesia 2014, for providing information on ethical clearance of the original study.

CONFLICTS OF INTEREST

The authors have completed and submitted the ICMJE Form for Disclosure of Potential Conflicts of Interest and none was reported.

\section{FUNDING}

This manuscript forms part of the PhD study of the first author. The present study was supported by the Indonesia Endowment Fund for Education (LPDP) (Grant number: S-2983/LPDP.3/2014).

PROVENANCE AND PEER REVIEW

Not commissioned; externally peer reviewed. 